\title{
Association between Alcoholism Family History and Alcohol Screening Scores among Alcohol-dependent Patients
}

\author{
So-Hyun Lee', Boung Chul Lee ${ }^{2,3}$, Jee Wook Kim, ${ }^{2,4}$ Jung Seo Yi', Ihn-Geun Choi ${ }^{1,2}$ \\ 'Department of Psychiatry, Hallym University Kangnam Sacred Heart Hospital, ${ }^{2}$ Hallym University Burn Institute, Hallym University, \\ ${ }^{3}$ Department of Psychiatry, Hallym University Hangang Sacred Heart Hospital, Seoul, ${ }^{4}$ Department of Psychiatry, Hallym University Dongtan \\ Sacred Heart Hospital, Hwaseong, Korea
}

\begin{abstract}
Objective: Several tests can be used to screen for alcohol dependence (AD), a prevalent disease with a heterogeneous etiology. As some patients with $A D$ have a strong familial tendency in this regard, a family history of alcohol use disorders can affect the outcomes of screening tests and diagnostic evaluations for AD. In this study, we evaluated associations between a family history of alcohol use disorders and evaluations using the Cut down, Annoyed, Guilty, Eye-opener (CAGE) test, Alcohol Use Disorder Identification Test (AUDIT), and Diagnostic and Statistical Manual of Mental Disorders-fourth edition (DSM-IV) diagnostic criteria among patients with $A D$.

Methods: We recruited 487 male patients with AD from eight hospitals in Korea. Patients were evaluated using the CAGE, AUDIT, and DSM-IV diagnostic criteria. Patients with and without family histories were compared in terms of these assessment tools. Results: Drinking initiation, uncontrollable drinking, and problem drinking occurred earlier and CAGE "annoyed" scores were higher in patients with a family history. Alcohol problems before the age of 25 years, frequency of spontaneous or compulsive alcohol-seeking behavior, and frequencies of psychological dependence and guilt related to alcohol use were also higher. Conclusion: Earlier drinking problems, higher scores on specific items of the CAGE, and AUDIT, and meeting more diagnostic criteria indicate more dependent, harmful drinking by patients with $A D$ who have a family history of this condition. Clinicians should consider patients' family history of alcohol use disorders when screening for AD to identify the correct diagnosis and develop appropriate treatment plans for these patients.
\end{abstract}

KEY WORDS: Alcohol use disorders; Family history; Cut down, Annoyed, Guilty, Eye-opener test; Alcohol Use Disorder Identification Test; Diagnostic and Statistical Manual of Mental Disorder-fourth edition.

\section{INTRODUCTION}

According to a survey on mental disorders conducted by the Korean Ministry of Health and Welfare, the lifetime prevalence of alcohol use disorders, including alcohol dependence (AD) and abuse, is $13.4 \%$ among the Korean population, which makes it the single most prevalent mental disorder. Specifically, one of five male adults $(20.7 \%)$ has suffered from an alcohol use disorder more than once. ${ }^{1)}$ Excessive drinking damages health and leads to economic losses, which have estimated at up to 15 trillion KRW (13.9 billion USD) per year in Korea. In this con-

\footnotetext{
Received: April 30, 2013 / Revised: June 19, 2013

Accepted: July 1, 2013

Address for correspondence: Ihn-Geun Choi, MD, PhD

Department of Psychiatry, Hallym University Kangnam Sacred Heart Hospital, 1 Shingil-ro, Yeongdeungpo-gu, Seoul 150-950, Korea Tel: +82-2-829-5187, Fax: +82-2-831-2554 E-mail: inngeun@nate.com
}

text, alcohol drinking has become an important health issue, and preventing and treating alcohol-related problems have been emerged as a vital national task. ${ }^{2)}$ Early detection and effective and intensive treatment of those who are at risk from alcohol is a more effective approach to decreasing the damage from this condition than is trying to address the problem at a later stage. ${ }^{3)}$ Healthcare centers and educational institutions in Korea have recommended distributing self-report tests and screening instruments. ${ }^{4)}$ The tools most widely used in Korea to screen and diagnose alcohol use disorders include the Cut down, Annoyed, Guilty, Eye-opener (CAGE) test, ${ }^{5)}$ the Alcohol Use Disorder Identification Test (AUDIT) ${ }^{6}{ }^{6}$ the Michigan Alcoholism Screening Test, ${ }^{7}$ and the Diagnostic Statistical Manual of Mental Disorder fourth edition (DSM-IV) diagnostic criteria for alcohol use disorders. ${ }^{8}$

Alcohol use disorders are caused by interactions among genetic, social, and environmental factors. Genetic fac-

(c) This is an Open-Access article distributed under the terms of the Creative Commons Attribution Non-Commercial License (http://creativecommons.org/licenses/by-nc/3.0) which permits unrestricted non-commercial use, distribution, and reproduction in any medium, provided the original work is properly cited 
tors, in particular, are the most important risk factors for $\mathrm{AD}$, and these presumably account for $60 \%$ of the variance, with environmental factors accounting for $40 \%{ }^{9}$ Patients with AD with a family history of alcohol use disorders show severe physical problems and high levels of antisocial behavior due to their problematic use of alcohol. ${ }^{10)}$ However, no research has been published on how a family history of alcohol use disorders relates to each item on the screening tests or to each of the diagnostic criteria for $\mathrm{AD}$, and questions about whether patients with $\mathrm{AD}$ with a positive family history should be evaluated differently from patients without such a family history during the screening or diagnostic process remain unanswered.

The purpose of this study was to compare differences in data from items on the CAGE and the AUDIT and from the DSM-IV diagnostic criteria for AD according to family history of alcohol use disorders and to investigate how a family history of alcohol use disorders is related to the clinical characteristics of patients with AD.

\section{METHODS}

\section{Subjects}

The sample included male patients aged 20-80 years who met the DSM-IV diagnostic criteria for AD according to more than two psychiatrists at Hallym University Hangang Sacred Heart Hospital and seven other hospitals in Korea between March 2008 and January 2012. In total, 487 patients participated in the study after listening to a complete description of the study and voluntarily signing an informed consent form. The study was approved by the institutional review board of Hallym University Hangang Sacred Heart Hospital.

The exclusion criteria were: 1) substance use disorder involving a substance other than alcohol, nicotine, or caffeine; 2) current or past history of major psychiatric disorders, such as schizophrenia or mood disorders; 3 ) major medical conditions other than alcohol-related disorders; and 4) the presence of severe behavioral or communication problems that would make a clinical examination difficult.

\section{Research Methods and Tools}

We collected data on participants' demographic characteristics including age, level of education, marital status, occupation, and religion. Participants also responded to questions about their drinking history and completed the CAGE to examine age, problems, and other variables re- lated to drinking and the AUDIT to investigate alcohol consumption, harmful drinking, and hazardous drinking.

\section{Questionnaire about drinking history}

This self-report 16-item questionnaire examines personal characteristics related to drinking. It includes questions about the age at which various drinking habits emerged and the presence of alcohol use disorders in first-degree relatives (parents and siblings) of patients.

2. Cut down, Annoyed, Guilty, Eye-opener (CAGE) test This survey was developed by Ewing ${ }^{11)}$ in 1984. Individuals who endorse two items may have serious alcohol-related issues and should obtain a consultation. Individuals who endorse more than three items should receive clinical treatment or be hospitalized due to severe alcoholism.

\section{Alcohol Use Disorder Identification Test (AUDIT)}

This tool was developed by the World Health Organization (WHO) in 1989 and is used to identify individuals who may be in danger due to their drinking problems. The test consists of 10 questions that measure three domains: drinking frequency and amount, hazardous drinking, and harmful drinking. In 2001, WHO defined scores between 8 and 16 as indicative of dangerous drinking that requires simple advice, scores between 16 and 20 as indicative of the need for a simple consultation and continuous observation, and scores higher than 20 as indicative of the need for a special consultation and assessment for problem drinking.

One Korean study using the AUDIT categorized scores of 12-14 points as indicative of problem drinking, including physical and psychosocial problems; those of 15-25 points as indicative of an alcohol use disorder; and those of 26 points or more as indicative of AD. ${ }^{12)}$ Additionally, Jeon ${ }^{4)}$ proposed criteria that defined problematic drinking as 8-11 points for males and 4-11 points for females, harmful drinking as $12-25$ points (15-25 points for alcohol abuse), and $\mathrm{AD}$ as scores higher than 26.

\section{Statistical Analysis}

Baseline differences in demographic characteristics, responses to CAGE and AUDIT items, and endorsement of diagnostic criteria between patients with a family history of alcohol use disorders in first-degree relatives (parents and siblings) and without a family history were compared using t-tests for continuous variables and $\chi^{2}$ tests for categorical variables. The data are summarized as mean \pm standard deviation (SD) after normality was evaluated us- 
ing Levene's test. Correlation coefficients were used to investigate correlations involving drinking history, including family history, the CAGE, the AUDIT, and the diagnostic criteria.

As the correlation analyses revealed several associations between demographic characteristics, items on the CAGE and AUDIT, and diagnostic criteria, on the one hand, and age, on the other, a multinomial logistic regression analysis for categorical variables and a univariate general linear model for continuous variables were used to correct for the confounding effects of age.

Data were analyzed using the PASW Statistics software (ver. 18.0; IBM Co., Armonk, NY, USA). Two-sided $\alpha$ $=0.05$ was considered to indicate statistical significance.

\section{RESULTS}

\section{Patient Characteristics}

According to the data, 141 patients with AD had a fam- ily history of alcohol use disorders, whereas 346 did not. The average age of patients with a family history was 46.08, whereas it was 48.93 in patients with no family history $(\mathrm{t}(480)=3.208, p=0.001)$. Patients with a family history started drinking at a younger age $(\mathrm{t}(479)=2.556$, $p=0.001)$ and reported drinking dyscontrol $(\mathrm{t}(449)=2.568$, $p=0.012)$ and behavioral problems during a drunken state $(\mathrm{t}(382)=3.418, p=0.001)$ at earlier ages than did patients with no family history. However, we found no significant difference between the groups in terms of educational level, marital status, occupation, religion, or smoking (Table 1).

\section{CAGE Items in Relation to Family History of Alcohol Use Disorder}

We compared patients with AD with and without a family history in terms of their responses to each item on the CAGE. Patients with a family history had significantly higher scores on "annoyed" than did patients with no family history $(\mathrm{t}(1)=4.659, p=0.031)$, and the average total

Table 1. Demographic and clinical characteristics of patients with alcohol dependence (AD) with and without a family history (FH) of alcohol use disorders

\begin{tabular}{|c|c|c|c|c|c|}
\hline Characteristic & $\begin{array}{l}\text { AD patients with } \mathrm{FH} \\
\qquad(n=141)\end{array}$ & $\begin{array}{l}\text { AD patients without FH } \\
\qquad(n=346)\end{array}$ & t or $\chi^{2}$ & df & $p$ value \\
\hline Age (year) & $46.08 \pm 8.38$ & $48.93 \pm 8.97$ & 3.208 & 480 & 0.001 \\
\hline Education (year) & $11.18 \pm 3.28$ & $11.68 \pm 3.18$ & 1.322 & 344 & 0.187 \\
\hline Marital status & & & 2.235 & 2 & 0.327 \\
\hline Never married & $41 / 111$ (35.3) & $76 / 272(27.9)$ & & & \\
\hline Married & $38 / 111(32.8)$ & $104 / 272(38.2)$ & & & \\
\hline Separated/divorced & $37 / 111(31.9)$ & $92 / 272(33.8)$ & & & \\
\hline Living with children & $69 / 115(60.6)$ & $181 / 271(66.8)$ & 1.631 & 1 & 0.202 \\
\hline Employed & $39 / 115(34.2)$ & $101 / 272(37.3)$ & 0.324 & 1 & 0.569 \\
\hline Smoking status & $59 / 63(93.7)$ & $149 / 169(88.2)$ & 1.489 & 1 & 0.222 \\
\hline Religious affiliation & $40 / 92(43.5)$ & $103 / 219(47.0)$ & 0.329 & 1 & 0.566 \\
\hline Comorbid illness & $59 / 110(53.6)$ & $135 / 260(51.9)$ & 0.091 & 1 & 0.763 \\
\hline Age at drinking onset (year) & $17.90 \pm 5.43$ & $19.29 \pm 5.40$ & 2.556 & 479 & $0.001^{*}$ \\
\hline Age at uncontrolled drinking (year) & $30.99 \pm 9.41$ & $33.53 \pm 9.47$ & 2.568 & 449 & $0.012^{*}$ \\
\hline Age at problem drinking (year) & $30.31 \pm 8.87$ & $33.74 \pm 9.23$ & 3.418 & 382 & $0.001^{*}$ \\
\hline
\end{tabular}

Values are presented as mean \pm standard deviation or number (\%)

${ }^{*}$ Corrected for confounding variable, age.

df, degree of freedom.

Table 2. CAGE scores of patients with alcohol dependence (AD) with and without a family history (FH) of alcohol use disorders

\begin{tabular}{|c|c|c|c|c|c|}
\hline CAGE score & $\begin{array}{l}\text { AD patients with } \mathrm{FH} \\
\qquad(\mathrm{n}=141)\end{array}$ & $\begin{array}{l}\text { AD patients without FH } \\
\qquad(n=346)\end{array}$ & t or $\chi^{2}$ & df & $p$ value \\
\hline Cut down & 135/141 (95.7) & $331 / 345(95.9)$ & 0.010 & 1 & 0.921 \\
\hline Annoyed & $125 / 141$ (88.5) & $277 / 344(80.5)$ & 4.659 & 1 & $0.031^{*}$ \\
\hline Guilty & $126 / 141(89.4)$ & $308 / 345(89.3)$ & 0.001 & 1 & $0.978^{*}$ \\
\hline Eye opener & $113 / 140(80.7)$ & $256 / 246(74.0)$ & 2.467 & 1 & 0.116 \\
\hline CAGE total & $3.54 \pm 0.76$ & $3.39 \pm 0.94$ & 1.863 & 316.9 & 0.063 \\
\hline
\end{tabular}

Values are presented as number (\%) or mean \pm standard deviation.

Cut down, cutting down on drinking; annoyed, annoyance at others' concern about drinking; guilty, feeling guilty about drinking; eye opener, using alcohol as an eye opener in the morning.

*Corrected for confounding variable, age.

df, degree of freedom. 
CAGE scores of patients with a family history were markedly higher than were those of patients with no family history $(\mathrm{t}(316.9)=1.863, p=0.063)$. The two patient groups did not significantly differ with regard to the other items (Table 2).

\section{AUDIT Items in Relation to Family History of Alcohol Use Disorder}

We compared patients with AD with and without a family history in terms of their responses to each AUDIT item. In particular, the scores of patients with a family history were significantly higher than were those without such a history on AUDIT items 4 (impaired control over drinking), 5 (increased salience of drinking), 6 (morning drinking), 8 (blackouts), and 9 (alcohol-related injuries). The average total AUDIT scores of patients with a family history were higher (mean=28.46, $\mathrm{SD}=7.04$ ) than were those of patients with no family history (mean $=25.70, \mathrm{SD}=7.30$; $\mathrm{t}(524)=4.094, p<0.001)$. The two groups did not differ significantly with respect to other items (Table 3 ).

\section{DSM-IV Diagnostic Criteria in Relation to Family History of Alcohol Use Disorder}

We compared patients with AD with and without a fam-

Table 3. AUDIT scores of patients with alcohol dependence (AD) with and without a family history (FH) of alcohol use disorders

\begin{tabular}{|c|c|c|c|c|c|}
\hline AUDIT score & $\begin{array}{l}\text { AD patients with } \mathrm{FH} \\
\qquad(n=141)\end{array}$ & $\begin{array}{l}\text { AD patients without FH } \\
\qquad(n=346)\end{array}$ & $t$ & df & $p$ value \\
\hline AUDIT 1 & $3.36 \pm 0.86$ & $3.31 \pm 0.84$ & 0.652 & 485 & 0.515 \\
\hline AUDIT 2 & $3.56 \pm 0.86$ & $3.44 \pm 0.90$ & 1.402 & 483 & $0.162^{*}$ \\
\hline AUDIT 3 & $3.33 \pm 0.88$ & $3.18 \pm 0.91$ & 1.577 & 484 & 0.115 \\
\hline AUDIT 4 & $2.82 \pm 1.13$ & $2.51 \pm 1.23$ & 2.503 & 794 & 0.016 \\
\hline AUDIT 5 & $2.49 \pm 1.24$ & $2.07 \pm 1.29$ & 3.311 & 481 & 0.001 \\
\hline AUDIT 6 & $2.44 \pm 1.33$ & $2.03 \pm 1.38$ & 3.011 & 485 & 0.002 \\
\hline AUDIT 7 & $2.66 \pm 1.22$ & $2.49 \pm 1.30$ & 1.306 & 484 & $0.192^{*}$ \\
\hline AUDIT 8 & $2.28 \pm 1.67$ & $1.85 \pm 1.20$ & 3.645 & 482 & $<0.001$ \\
\hline AUDIT 9 & $2.07 \pm 1.49$ & $1.64 \pm 1.67$ & 2.803 & 286.7 & $0.005^{*}$ \\
\hline AUDIT 10 & $3.46 \pm 1.07$ & $3.30 \pm 1.19$ & 1.454 & 288.8 & $0.147^{*}$ \\
\hline AUDIT total & $28.46 \pm 7.04$ & $25.70 \pm 7.30$ & 4.094 & 524 & $<0.001^{*}$ \\
\hline
\end{tabular}

AUDIT, Alcohol Use Disorder Identification Test: AUDIT 1, frequency of drinking: AUDIT 2, typical quantity; AUDIT 3, frequency of heavy drinking: AUDIT 4, impaired control over drinking; AUDIT 5, increased salience of drinking; AUDIT 6, morning drinking; AUDIT 7, guilt after dinking; AUDIT 8, blackouts; AUDIT 9, alcohol-related injuries; AUDIT 10, others concerned about drinking.

${ }^{*}$ Corrected for confounding variable, age.

df, degree of freedom.

Table 4. Endorsement of DSM-IV diagnostic criteria by patients with alcohol dependence (AD) with and without a family history (FH) of alcohol use disorders

\begin{tabular}{|c|c|c|c|c|c|}
\hline DSM-IV diagnostic criteria & $\begin{array}{l}\text { AD patients with } \mathrm{FH} \\
\qquad(n=141)\end{array}$ & $\begin{array}{l}\text { AD patients without FH } \\
\qquad(n=346)\end{array}$ & t or $\chi^{2}$ & $d f$ & $p$ value \\
\hline DSM-IV la & $107(75.9)$ & $202(58.4)$ & 13.236 & 1 & 0.001 \\
\hline DSM-IV 1b & $35(24.8)$ & $126(36.4)$ & 6.084 & 1 & 0.014 \\
\hline DSM-IV 2a & $68(48.2)$ & $147(42.5)$ & 1.139 & 1 & 0.247 \\
\hline DSM-IV 2b & $43(30.5)$ & $109(31.5)$ & 0.047 & 1 & 0.828 \\
\hline DSM-IV 3 & $107(75.9)$ & $224(64.7)$ & 5.717 & 1 & 0.017 \\
\hline DSM-IV 4 & $120(85.1)$ & $272(78.6)$ & 2.690 & 1 & $0.101^{*}$ \\
\hline DSM-IV 5 & $100(70.9)$ & $204(59.0)$ & 6.111 & 1 & 0.013 \\
\hline DSM-IV 6 & $115(81.6)$ & $229(66.2)$ & 11.418 & 1 & 0.001 \\
\hline DSM-IV 7 & $87(61.7)$ & $193(55.8)$ & 1.437 & 1 & $0.231^{*}$ \\
\hline DSM-IV total & $5.19 \pm 1.74$ & $4.87 \pm 1.92$ & 1.739 & 485 & 0.083 \\
\hline
\end{tabular}

Values are presented as mean \pm standard deviation or number (\%).

Dignostic Statistical Manual of Mental Disorder fourth edition (DSM-IV) 1, tolerance, as defined by either of the following: a) a need for markedly increased amounts of alcohol to achieve intoxication or desired effect, b) markedly diminished effect with continued use of the same amount of alcohol; DSM-IV 2, withdrawal, as defined by either of the following: a) the characteristic withdrawal syndrome for alcohol (refer to DSM-IV for further details), b) alcohol is taken to relieve or avoid withdrawal symptoms: DSM-IV 3 , alcohol is often taken in larger amounts or over a longer period than was intended; DSM-IV 4, persistent desire or unsuccessful efforts to cut down or control alcohol use; DSM-IV 5, a great deal of time spent in activities necessary to obtain alcohol, use alcohol or recover from its effects; DSM-IV 6 , important social, occupational, or recreational activities are given up or reduced because of alcohol use; DSM-IV 7, alcohol use is continued despite knowledge of having a persistent or recurrent physical or psychological problem that is likely to have been caused or exacerbated by the alcohol (e.g., continued drinking despite recognition that an ulcer was made worse by alcohol consumption); df, degree of freedom.

${ }^{*}$ Corrected for confounding variable, age. 
ily history in terms of the DSM-IV diagnostic criteria. Patients with a family history were more likely than those without such a history to endorse items 1a (need for markedly increased amounts of alcohol to achieve intoxication or desired effect), $1 b$ (markedly diminished effect with continued use of the same amount of alcohol), 3 (frequent consumption of alcohol in larger amounts or over a longer period than was intended), 5 (a great deal of time spent engaging in activities necessary to obtain alcohol, use alcohol, or recover from its effects), and 6 (abandonment of or reduction in important social, occupational, or recreational activities because of alcohol use). The groups did not differ significantly in terms of other items (Table 4).

\section{DISCUSSION}

The purpose of this study was to investigate associations between a family history of alcohol use disorders and scores on the CAGE and AUDIT and endorsement of DSM-IV diagnostic criteria among alcohol-dependent patients.

Patients with AD with a family history obtained higher scores on the "annoyed" item of the CAGE, leading to higher total CAGE scores in this group than in patients with no family history. This suggests that patients with a family history of alcohol use disorders are exposed to a family environment in which family members are sensitive to and critical of the drinking of other family members, resulting in annoyance about being blamed for drinking. Patients with AD commonly use defense mechanisms involving projection, turning against others, and denial. These defenses are related to low levels of insight about drinking, ${ }^{13)}$ which renders the comments made by family members about drinking more annoying. Some studies have shown that AD leads to criticism and negative feelings among family members regarding alcohol-related issues, yielding decreased conversation, which interferes with the development of non-verbal social skills, including the ability to express emotions and rapidly respond to reactions from other people. ${ }^{14)}$ It has been reported that children raised in an environment without emotional support face a greater risk of abusing alcohol in adulthood. ${ }^{15)}$ This may be interpreted as evidence supporting the role of alcohol use disorders in the creation of an overall family atmosphere and as indicative of the harmful impact of these disorders on normative emotional development and problems related to alcohol use among children. These phenomena contribute to a vicious cycle in which the family history of alcoholism is perpetuated.
Thus, it would be useful to further investigate responses to the "annoyed" item among patients with AD with a family history of alcohol use disorders.

Patients with a family history of alcohol use disorders obtained higher scores on AUDIT items 4, 5, and 6, which inquired about the symptoms of $\mathrm{AD}$, on items 8 and 9, which addressed harmful drinking, and on the AUDIT as a whole. This may show that problems related to AD and harmful drinking are more strongly related to family history than are those related to hazardous drinking. This is consistent with studies concluding that a family history of alcohol use disorders is related to the development and chronicity of AD. ${ }^{16,17)}$ The AUDIT is more accurate and effective than other screening tests in distinguishing harmful drinking and alcohol use disorder, with a sensitivity of $51-97 \%$ and a specificity of $78-96 \% .{ }^{18)}$ However, it is difficult to use for screening in primary care setting because it includes 10 questions, is complex, and takes a long time to answer. Many studies have focused on developing shorter tests summarizing the AUDIT items in the service of simple and easy application. Brief versions of AUDIT include the AUDIT-C, ${ }^{19)}$ which includes items 1-3, and the AUDIT-PC ${ }^{20)}$ with AUDIT items 1, 2, 4, 5, and 10. Additionally, AUDIT-3, ${ }^{21)}$ with AUDIT item 3 alone, and AUDIT-5, ${ }^{22)}$ with AUDIT items 2, 3, 5, 9, and 10 , are widely used in clinical settings. However, these abbreviated versions of the AUDIT usually consist of questions related to hazardous alcohol use and are more useful in screening for less severe hazardous drinkers than for severe harmful alcohol users. ${ }^{23)}$ Furthermore, scores on the dependence subscale of the AUDIT may increase the likelihood of meeting criteria for a diagnosis of AD. ${ }^{24)}$ When using a brief version of the AUDIT with patients with a family history of alcohol use disorders, we should not overlook the risk of neglecting factors related to $\mathrm{AD}$ and harmful drinking.

In this study, the DSM-IV diagnostic criteria include more items related to tolerance for alcohol-dependent patients with a family history, including drinking more than before and drinking more than expected or longer than intended, compared with patients with no family history. Furthermore, patients with a family history devote more time to drinking, which leads them to give up or reduce important social, occupational, and recreational activities. It may be inferred that patients with $\mathrm{AD}$ with a family history are used to watching other family members drink at home and that this accustoms them to drinking, which leads to excessive drinking and failure in the performance of other activities. 
The limitations of this study include difficulties generalizing results for hospitalized patients to all patients with AD. The reliability of the results may be increased by recruiting patients from various treatment settings and the community. Additionally, self-report screening tests are limited due their use of subjective methods and the possible difficulties experienced by respondents in understanding the questions. Moreover, participants may provide unrealistically positively responses to such questionnaires, under-reporting the severity of their condition by minimizing or denying their symptoms due to poor insight and use of defense mechanisms such as denial and projection.

$\mathrm{AD}$ is a heterogeneous disease. Many researchers have tried to examine and identify subtypes of AD to determine the causes of and treatments for each clinical subtype. Understanding the various subtypes of AD could help in providing individualized treatment for patients. Data on a family history of alcohol use disorders obtained from the CAGE and AUDIT and use of the diagnostic criteria for $\mathrm{AD}$ may be useful for subtyping and individualizing the treatment of AD.

This study found several differences between patients with $\mathrm{AD}$ with and without a family history of alcohol use disorder in terms of responses to the CAGE, AUDIT, and the DSM-IV diagnostic criteria: the data showed increased annoyance, AD symptoms, harmful drinking, and tolerance and reduced recreational and social activities among patients with AD with a family history of alcohol use disorders. The earlier drinking problems, higher scores on specific items of the CAGE and AUDIT, and endorsement of more diagnostic criteria among patients with $\mathrm{AD}$ with a family history imply greater dependence and more harmful drinking in these patients than in patients with AD with no family history. Thus, these characteristics should be considered in the process of screening, diagnosing, and treating patients with $\mathrm{AD}$ with a family history of alcohol use disorder. Clinicians should consider a family history of alcohol use disorders in the process of screening for and diagnosing AD to facilitate the appropriate assessment and treatment of these patients.

\section{acknowledgments}

This study was supported by a grant of the Korea Healthcare Technology R\&D Project, Ministry for Health, Welfare and Family Affairs, Republic of Korea (grant number A084589). SHL, BCL, JWK, JSY and ICG designed this study. All authors contributed to the data collection, participated in writing the manuscript and all agreed to accept equal responsibility for the content of the paper.

\section{REFERENCES}

1. Ministry of Health and Welfare (Korea). The Epidemiological Survey of Mental Disorders in Korea. 2012 [cited 2013 Apr 25]. Available from http://www.mw.go.kr/front_new/jb/ sjb030301vw.jsp?PAR_MENU_ID=03\&MENU_ID=031604 $\&$ CONT_SEQ $=274852$ \&page $=1$

2. Lee S, Chung W, Kim IS, Kim HJ, Cho W, Shin E, et al. Socioeconomic costs of alcohol drinking in Korea. J Korean Acad Fam Med 2008;29:201-212.

3. Moyer A, Finney JW, Swearingen CE, Vergun P. Brief interventions for alcohol problems: a meta-analytic review of controlled investigations in treatment-seeking and nontreatment-seeking populations. Addiction 2002;97:279-292.

4. Jeon Y. Selecting alcohol use disorder and early involvement for the primary medical institutions and mental health personnel. Seoul:Korean Alcohol Research Foundation;2006.

5. Mayfield D, McLeod G, Hall P. The CAGE questionnaire: validation of a new alcoholism screening instrument. Am J Psychiatry 1974;131:1121-1123.

6. Saunders JB, Aasland OG, Babor TF, de la Fuente JR, Grant M. Development of the Alcohol Use Disorders Identification Test (AUDIT): WHO collaborative project on early detection of persons with harmful alcohol consumption-II. Addiction 1993;88:791-804.

7. Selzer ML. The Michigan alcoholism screening test: the quest for a new diagnostic instrument. Am J Psychiatry 1971;127:1653-1658.

8. American Psychiatric Association. Diagnostic and statistical manual of mental disorder fourth edition (DSM-IV). Washington, DC:American Psychiatric Association;1994.

9. Sadock BJ, Kaplan HI, Sadock VA. Kaplan \& Sadock's synopsis of psychiatry. Philadelphia:Lippincott Williams \& Wilkins;2007.

10. Johnson EO, Pickens RW. Familial transmission of alcoholism among nonalcoholics and mild, severe, and dyssocial subtypes of alcoholism. Alcohol Clin Exp Res 2001;25:661666.

11. Ewing JA. Detecting alcoholism. The CAGE questionnaire. JAMA 1984;252:1905-1907.

12. Kim JS, Oh MK, Park BK, Lee MK, Kim GJ. Screening criteria of alcoholism by alcohol use disorders identification test (AUDIT) in Korea. J Korean Acad Fam Med 1999;20: 1152-1159.

13. Rohsenow DJ, Erickson RC, O'Leary MR. The defense mechanism inventory and alcoholics. Int J Addict 1978;13: 403-414.

14. Jones DC, Houts R. Parental drinking, parent-child сотmunication, and social skills in young adults. J Stud Alcohol 1992;53:48-56.

15. Shaw BA. Lack of emotional support from parents early in life and alcohol abuse later in life. Int J Aging Hum Dev 2006;63:49-72.

16. Hasin D, Paykin A, Endicott J. Course of DSM-IV alcohol dependence in a community sample: effects of parental history and binge drinking. Alcohol Clin Exp Res 2001;25:411414.

17. Schuckit MA, Tsuang JW, Anthenelli RM, Tipp JE, Nurnberger JI Jr. Alcohol challenges in young men from alcoholic pedigrees and control families: a report from the COGA project. J Stud Alcohol 1996;57:368-377. 
18. Fiellin DA, Reid MC, O'Connor PG. Outpatient management of patients with alcohol problems. Ann Intern Med 2000;133:815-827.

19. Bush K, Kivlahan DR, McDonell MB, Fihn SD, Bradley KA. The AUDIT alcohol consumption questions (AUDIT-C): an effective brief screening test for problem drinking. Ambulatory Care Quality Improvement Project (ACQUIP). Alcohol Use Disorders Identification Test. Arch Intern Med 1998;158:1789-1795.

20. Piccinelli M, Tessari E, Bortolomasi M, Piasere O, Semenzin M, Garzotto N, et al. Efficacy of the alcohol use disorders identification test as a screening tool for hazardous alcohol intake and related disorders in primary care: a validity study. BMJ 1997;314:420-424.

21. Gordon AJ, Maisto SA, McNeil M, Kraemer KL, Conigliaro
RL, Kelley ME, et al. Three questions can detect hazardous drinkers. J Fam Pract 2001;50:313-320.

22. Kim JW, Lee BC, Lee DY, Seo CH, Kim S, Kang TC, et al. The 5-item Alcohol Use Disorders Identification Test (AUDIT-5): an effective brief screening test for problem drinking, alcohol use disorders and alcohol dependence. Alcohol Alcohol 2013;48:68-73.

23. Aertgeerts B, Buntinx F, Ansoms S, Fevery J. Screening properties of questionnaires and laboratory tests for the detection of alcohol abuse or dependence in a general practice population. Br J Gen Pract 2001;51:206-217.

24. Johnson JA, Lee A, Vinson D, Seale JP. Use of AUDITbased measures to identify unhealthy alcohol use and alcohol dependence in primary care: a validation study. Alcohol Clin Exp Res 2013;37(Suppl 1):E253-E259. 